Editorial

\title{
Prevención y control de la tuberculosis en pueblos indígenas de la Región de las Américas
}

\section{Tuberculosis prevention and control in indigenous populations in the Region of the Americas}

\author{
Norman Gil, Pedro Avedillo, Rafael López ${ }^{1}$ \\ Sandra del Pino ${ }^{2}$
}

${ }^{1}$ Organización Panamericana de la Salud, Departamento de Enfermedades Transmisibles y Determinantes Ambientales de la Salud, Unidad de VIH, Hepatitis, Tuberculosis e Infecciones de Transmisión Sexual. Washington, D.C., EE.UU

${ }^{2}$ Organización Panamericana de la Salud, Oficina de Equidad, Género y Diversidad Cultural. Washington, D.C., EE.UU

La tuberculosis (TB), contrario a lo que se creyó durante mucho tiempo, está presente en la Región de las Américas desde antes de la colonización. Sin embargo, fue durante el proceso de asentamiento de los europeos cuando alcanzó niveles epidémicos, al transmitirse rápidamente entre poblaciones indígenas, donde contribuyeron a su diseminación factores como la marginación social, la inseguridad alimentaria y otras comorbilidades. Siglos después, la TB continúa siendo una enfermedad de gran importancia sanitaria a nivel mundial y regional que afecta desproporcionadamente a las poblaciones identificadas como especialmente vulnerables para infectarse y enfermar, entre ellas los pueblos indígenas.

En las Américas la TB afecta a los pueblos indígenas de forma particular, registrando en la última década tasas de incidencia que superan en gran medida a las de la población general; por ejemplo, se observó en el año 2016 que la probabilidad de padecer TB en una persona autoidentificada como indígena en Paraguay era nueve veces mayor que en personas no indígenas; y en países como Brasil, Colombia, Panamá y Venezuela (República Bolivariana de) esta probabilidad fue de 2 a 3 veces más ${ }^{(1)}$. A nivel más específico, hay casos como el de los pueblos Aché de Paraguay y Yanomami en la Amazonia brasileña, donde la incidencia de TB registrada llega a ser 75 y 35 veces mayor que en la población no indígena de cada país respectivamente ${ }^{(1)}$.

Este es un artículo de acceso abierto, distribuido bajo los términos de Licencia de Atribución Creative Commons, que permite el uso, distribución y reproducción en cualquier medio, siempre que se acredite el origen y la fuente originales. Como citar este artículo: Gil $N$, Avedillo P, López R, Pino S del. Prevención y Control de la Tuberculosis en Pueblos Indígenas de la Región de las Américas. (Editorial). Rev. Nac. (Itauguá). 2021;13(2):001-004. 
Los Estados miembros de la Organización Panamericana de la Salud (OPS) reconocieron la necesidad de contar con enfoques diferenciados para abordar la situación de salud de los pueblos indígenas, las poblaciones afrodescendientes y otros grupos étnicos al adoptar, de forma unánime, la primera Política sobre Etnicidad y Salud en 2017. En sintonía con este compromiso y para hacer frente a la situación de TB en las poblaciones indígenas, la OPS ha elaborado los "Lineamientos para la prevención y el control de la tuberculosis en los pueblos indígenas de la región de las Américas", documento que sirve como herramienta práctica para ayudar a implementar la Estrategia Fin de la $T B^{(2)}$, que orienta el abordaje de la TB en el mundo, desde un enfoque intercultural que va en consonancia con las líneas prioritarias tanto de la Política sobre Etnicidad y Salud, como de su Estrategia y Plan de Acción 2019 - 2025 $5^{(3,4)}$.

Los lineamientos recogen la experiencia acumulada de la OPS y las buenas prácticas de algunos de sus Estados Miembros en los últimos años, e incorporan las discusiones y experiencias compartidas en reuniones regionales celebradas sobre el tema, haciendo hincapié en la innovación y la inclusión social. Este enfoque sugiere cambiar los paradigmas convencionales que promueven algunas acciones específicas para reducir gradualmente la incidencia de la TB, por acciones multisectoriales intensivas que han demostrado ser más eficaces para acelerar la disminución de la epidemia de $\mathrm{TB}^{(1)}$. Enfrentar la TB en los pueblos indígenas de la Región se convierte en un reto y en una oportunidad para intervenir una patología en la cual es importante el abordaje integral de los factores que la determinan en un marco sociocultural específico, permitir el fortalecimiento de los sistemas de salud tanto occidentales como tradicionales y aportar en promoción y defensa de los derechos de las minorías étnicas.

Para una adecuada implementación de los lineamientos es fundamental la vinculación de diversos actores que trabajan de forma coordinada con los líderes y lideresas representantes de pueblos indígenas de los niveles nacionales y subnacionales; entre estos actores destacan: las autoridades nacionales, subnacionales y locales del sector de la salud; jefes de programas nacionales y subnacionales de TB y sus equipos técnicos; los responsables de asuntos indígenas y étnicos en los ministerios de salud u otros ministerios; personal del nivel local que preste atención en salud a poblaciones indígenas; grupos de personas afectadas con TB en zonas indígenas; sociedad civil organizada y organizaciones no gubernamentales que trabajen o vayan a trabajar en favor de la población indígena; investigadores, docentes y potenciales donantes; entre otros ${ }^{(1)}$. 
En la actualidad Paraguay es uno de los países pioneros en la implementación de los lineamientos. Desde el 2019, el país ha trabajado en la ejecución de los diferentes pasos que sugieren los lineamientos y que deben tenerse en cuenta en el desarrollo operativo de la implementación inicial de los mismos. El departamento de Caaguazú fue priorizado para estas acciones, y en él, específicamente el municipio de Repatriación, el pueblo indígena Mbya Guaraní y la comunidad de Pindo'i.

Paraguay en el contexto de la pandemia por la COVID-19, no ha sido ajeno al impacto que ésta ha generado dentro de los sistemas de salud en todo el mundo, especialmente los relacionados con interrupciones en el acceso a la atención de las personas afectadas por la TB. La Organización Mundial de la Salud (OMS) ha indicado en un informe preliminar, que en el 2020 se dio una reducción del $21 \%$ en el número de personas que recibieron atención por TB en el mundo comparado con 2019 y que, además, se estimó que estas interrupciones podrían causar medio millón de muertes más entre los afectados por la $\mathrm{TB}^{(5)}$. En este sentido, es lógico pensar en un fuerte impacto al interior de los pueblos indígenas, si consideramos que la situación de invisibilidad y exclusión que enfrentan estas poblaciones representa un reto para alcanzar las metas de los Objetivos de Desarrollo Sostenible (ODS) relacionados con la salud, como las referentes al acceso universal a la salud y la cobertura universal de salud, la TB, la malaria y la salud mental, entre otras ${ }^{(3)}$.

Sin embargo, un importante y reciente logro del país es el Proyecto de Ley "Que establece los derechos y obligaciones en la prevención, atención integral de la salud y protección social de las personas afectadas por la tuberculosis" recientemente sancionado por la cámara de Diputados, el cual es un potente marco normativo que se convierte en una gran oportunidad para el Programa Nacional de Control de Tuberculosis (PNCT) y el sistema de salud en general, para revertir los efectos producidos por la pandemia. Además, brinda un adecuado soporte para la implementación de los "Lineamientos para la prevención y el control de la TB en los pueblos indígenas de la región de las Américas" al interior del país, lo que permitirá avanzar hacia la futura eliminación de esta enfermedad que se ubica entre las primeras causales de muerte, no solo en Paraguay sino en el mundo entero.

Durante el 2021 desde la oficina Regional de la OPS, se están realizado visitas virtuales de seguimiento a los países que iniciaron en 2019 la implementación inicial de los lineamientos regionales y se cuenta con la firme decisión de avanzar con otros países de la región en este proceso antes de finalizar el año. 
Como reza el slogan actual de la lucha mundial contra la tuberculosis "El tiempo corre", necesitamos actuar.

\title{
Norman Gil $\quad$ Pedro Avedillo $\quad$ Rafael López
}

Organización Panamericana de la Salud, Departamento de Enfermedades Transmisibles y Determinantes Ambientales de la Salud, Unidad de VIH, Hepatitis, Tuberculosis e Infecciones de Transmisión Sexual.

\author{
Sandra del Pino \\ Organización Panamericana de la Salud \\ Oficina de Equidad, Género y Diversidad Cultural
}

\section{REFERENCIAS}

1. Organización Panamericana de la Salud. Lineamientos para la prevención y el control de la tuberculosis en los pueblos indígenas de la Región de las Américas. [Internet]. Washington, D.C.: OPS; 2020. Disponible en: https://iris.paho.org/handle/10665.2/53308.

2. Organización Mundial de la Salud. Implementación de la estrategia fin de la TB: aspectos esenciales [Internet]. $\quad 2016 . \quad$ OMS; Disponible https://www.paho.org/es/documentos/implementacion-estrategia-fin-tb-aspectos-esenciales-2016.

3. Organización Panamericana de la Salud. Política sobre etnicidad y salud [Internet]. 29a Conferencia Sanitaria Panamericana; 25 al 29 de septiembre de 2017; Washington DC. Washington DC: OPS; $2017 \quad$ (CSP29/7, Rev. 1). Disponible en: https://www.paho.org/hq/index.php?option=com_docman\&view=download\&category_slug=29es-9250\&alias=42014-csp29-7-s-014\&Itemid=270\&lang=es.

4. Organización Panamericana de la Salud. Estrategia y plan de acción sobre etnicidad y salud 20192025 [Internet]. Washington, D.C.; 2019. Disponible en: https://www.paho.org/hq/index.php?option=com_docman\&view=download\&category_slug=29es-9250\&alias=42014-csp29-7-s-014\&Itemid=270\&lang=es.

5. Organización Mundial de la Salud. Impact of the COVID-19 pandemic on TB detection and mortality in 2020 [Internet]. 2021. Disponible en: https://www.who.int/publications/m/item/impactof-the-covid-19-pandemic-on-tb-detection-and-mortality-in-2020

NOTA: Si bien los autores son funcionarios de la Organización Panamericana de la Salud, las opiniones expresadas en esta publicación son de su exclusiva responsabilidad y no representan necesariamente las decisiones ni las políticas de la Organización Panamericana de la Salud. 